General Mathematics Vol. 27, No. 2 (2019), 43-57

DOI: $10.2478 / \mathrm{gm}-2019-0014$

Sciendo

\title{
Univalence Criteria for a General Integral Operator ${ }^{1}$
}

\author{
Camelia Bărbatu, Daniel Breaz
}

\begin{abstract}
In this paper we introduce a new general integral operator for analytic functions in the open unit disk $\mathbb{U}$ and we obtain sufficient conditions for univalence of this integral operator.
\end{abstract}

2010 Mathematics Subject Classification: 30C45, 30C75.

Key words and phrases: Integral operator; analytic and univalent functions; unit disk.

\section{Introduction}

Let $\mathcal{A}$ denote the class of the functions of the form:

$$
f(z)=z+\sum_{n=2}^{\infty} a_{n} z^{n}
$$

which are analytic in the open unit disk

$$
\mathbb{U}=\{z \in \mathbb{C}:|z|<1\}
$$

and satisfy the following usual normalization conditions:

$$
f(0)=f^{\prime}(0)-1=0,
$$

\footnotetext{
${ }^{1}$ Received 2 June, 2019

Accepted for publication (in revised form) 12 December, 2019
} 
$\mathbb{C}$ being the set of complex numbers.

We denote by $\mathcal{S}$ the subclass of $\mathcal{A}$ consisting of functions $f \in \mathcal{A}$, which are univalent in $\mathbb{U}$.

A function $f \in \mathcal{A}$ is said to belong to the class $\mathcal{R}(\lambda), 0 \leq \lambda<1$, if

$$
\operatorname{Re}\left[f^{\prime}(z)\right]>\lambda, \quad z \in \mathbb{U} .
$$

We denote by $\mathcal{P}$ the class of the functions $p(z)$ which are analytic in $\mathbb{U}$ and satisfy the following conditions:

$$
p(0)=1, \quad \operatorname{Re}(p(z))>0, \quad z \in \mathbb{U} .
$$

We consider the integral operator:

$$
\mathcal{C}_{n}(z)=\left\{\delta \int_{0}^{z} t^{\delta-1} \coprod_{i=1}^{n}\left[\left(\frac{f_{i}(t)}{t} e^{g_{i}(t)}\right)^{\alpha_{i}-1}\left(h_{i}{ }^{\prime}(t)\right)^{\beta_{i}}\left(\frac{\left.h_{i}(t)\right)}{t}\right)^{\gamma_{i}}\right] \mathrm{dt}\right\}^{\frac{1}{\delta}}
$$

where $f_{i}, g_{i}, h_{i}$ are analytic in $\mathbb{U}$ and $\delta, \alpha_{i}, \beta_{i}, \gamma_{i}$ are complex numbers for all $i=\overline{1, n}$, $n \in \mathbb{N} \backslash\{0\}, \delta \in \mathbb{C}$, with $\operatorname{Re} \delta>0$..

This is a general integral operator of Pfaltzgraff, Kim-Merkes and Oversea types which extends also the other operators as follows:

Remark 1.1. i) For $n=1, \delta=1$ and $\alpha_{1}-1=\beta_{1}=0$ we obtain the integral operator which was studied by Kim-Merkes [7]

$$
\mathcal{F}_{\alpha}(z)=\int_{0}^{z}\left(\frac{f(t)}{t}\right)^{\alpha} d t
$$

ii) For $n=1, \delta=1$ and $\alpha_{1}-1=\gamma_{1}=0$ we obtain the integral operator which was studied by Pfaltzgraff [18]

$$
\mathcal{G}_{\alpha}(z)=\int_{0}^{z}\left(f^{\prime}(t)\right)^{\alpha} d t .
$$

iii) For $\alpha_{i}-1=\beta_{i}=0$ we obtain the integral operator which was defined and studied by D. Breaz and N. Breaz [2]

$$
\mathcal{D}_{n}(z)=\left[\delta \int_{0}^{z} t^{\delta-1} \coprod_{i=1}^{n}\left(\frac{f_{i}(t)}{t}\right)^{\alpha_{i}} d t\right]^{\frac{1}{\delta}},
$$

this integral operator is a generalization of the integral operator introduced by Pascu and Pescar [12].

iv) For $\alpha_{i}-1=\gamma_{i}=0$ we obtain the integral operator which was defined and studied by D. Breaz, Owa and N. Breaz [4]

$$
\mathcal{I}_{n}(z)=\left[\delta \int_{0}^{z} t^{\delta-1} \coprod_{i=1}^{n}\left[f_{i}^{\prime}(t)\right]^{\alpha_{i}} d t\right]^{\frac{1}{\delta}}
$$


this integral operator is a generalization of the integral operator introduced by Pescar and Owa in [17].

v) For $\alpha_{i}-1=0$ we obtain the integral operator which was defined and studied by Frasin [5]

$$
\mathcal{F}_{n}(z)=\left[\delta \int_{0}^{z} t^{\delta-1} \coprod_{i=1}^{n}\left(\frac{f_{i}(t)}{t}\right)^{\alpha_{i}}\left(f_{i}{ }^{\prime}(t)\right)^{\beta_{i}} d t\right]^{\frac{1}{\delta}},
$$

this integral operator is a generalization of the integral operator introduced by Oversea in [9].

vi) For $n=1, \delta=\beta$ and $\alpha_{i}-1=\alpha_{i}$ and $\beta_{i}=\gamma_{i}=0$ we obtain the integral operator which was defined and studied by Stanciu in [19]

$$
\mathcal{H}_{1}(z)=\left[\beta \int_{0}^{z} t^{\beta-1}\left(\frac{f(t)}{t} e^{g(t)}\right)^{\alpha} d t\right]^{\frac{1}{\beta}} .
$$

Thus, the integral operator $\mathcal{C}_{n}$, introduced here by the formula (2), can be considered as an extension and a generalization of these operators above mentioned.

We need in our present investigation the following lemmas:

Lemma 1.2. (Pascu [11]) Let $\gamma, \delta$ be complex numbers, Re $\gamma>0$ and $f \in \mathcal{A}$. If

$$
\frac{1-|z|^{2 \operatorname{Re} \gamma}}{\operatorname{Re} \gamma}\left|\frac{\left.z f^{\prime \prime}(z)\right)}{f^{\prime}(z)}\right| \leq 1,
$$

for all $z \in \mathbb{U}$, then for any complex number $\delta$, Re $\delta \geq \operatorname{Re} \gamma$, the function $F_{\delta}$ defined by

$$
F_{\delta}(z)=\left(\delta \int_{0}^{z} t^{\delta-1} f^{\prime}(t) d t\right)^{\frac{1}{\delta}},
$$

is regular and univalent in $\mathcal{U}$.

Lemma 1.3. (Pescar [14]) Let $\delta$ be complex number, Re $\delta>0$ and $c$ a complex number, $|c| \leq 1, c \neq-1$, and $f \in \mathcal{A}, f(z)=z+a_{2} z^{2}+\ldots$. If

$$
\left.|c| z\right|^{2 \delta}+\left(1-|z|^{2 \delta}\right) \frac{\left.z f^{\prime \prime}(z)\right)}{\delta f^{\prime}(z)} \mid \leq 1,
$$

for all $z \in \mathbb{U}$, then the function $F_{\delta}$ defined by

$$
F_{\delta}(z)=\left(\delta \int_{0}^{z} t^{\delta-1} f^{\prime}(t) d t\right)^{\frac{1}{\delta}}
$$

is regular and univalent in $\mathcal{U}$. 
Lemma 1.4. (General Schwarz Lemma $[8]$ ) Let $f$ be the function regular in the disk $\mathbb{U}_{R}=\{z \in \mathbb{C}:|z|<R, R>0\}$ with $|f(z)|<M$ for a fixed number $\mathrm{M}>0$ fixed. If $f(z)$ has one zero with multiplicity order bigger than a positive integer $m$ for $z=0$, then

$$
|f(z)| \leq \frac{M}{R^{m}} z^{m}, \quad z \in \mathbb{U}_{R}
$$

The equality for $z \neq 0$ can hold only if

$$
f(z)=e^{i \theta} \frac{M}{R^{m}} z^{m}
$$

where $\theta$ is constant.

\section{Main results}

Our main results give sufficient conditions for the general integral operator $\mathcal{C}_{n}$ defined by (2) to be univalent in the open disk $\mathbb{U}$.

Theorem 2.1. Let $\gamma, \delta, \alpha_{i}, \beta_{i}, \gamma_{i}$ be complex numbers, $c=$ Re $>0, M_{i}, N_{i}, P_{i}, Q_{i}$ are real positive numbers, $i=\overline{1, n}$, and $f_{i}, g_{i}, h_{i} \in \mathcal{A}, f_{i}(z)=z+a_{2 i} z^{2}+a_{3 i} z^{3}+\ldots$, $g_{i}(z)=z+b_{2 i} z^{2}+b_{3 i} z^{3}+\ldots ., h_{i}(z)=z+c_{2 i} z^{2}+c_{3 i} z^{3}+\ldots ., i=\overline{1, n}$. If

$$
\begin{gathered}
\left|\frac{z f_{i}^{\prime}(z)}{f_{i}(z)}-1\right| \leq M_{i}, \quad\left|g_{i}(z)\right| \leq N_{i}, \quad\left|\frac{z h_{i}^{\prime \prime}(z)}{h_{i}^{\prime}(z)}\right| \leq P_{i}, \\
\left|\frac{z h_{i}^{\prime}(z)}{h_{i}(z)}-1\right| \leq Q_{i}, \quad\left|\frac{z g_{i}^{\prime}(z)}{g_{i}(z)}\right| \leq 1,
\end{gathered}
$$

for all $z \in \mathbb{U}, i=\overline{1, n}$ and

$$
\sum_{i=1}^{n}\left[\left|\alpha_{i}-1\right|\left(M_{i}+N_{i}\right)+\left|\beta_{i}\right| P_{i}+\left|\gamma_{i}\right| Q_{i}\right] \leq \frac{(2 c+1)^{\frac{2 c+1}{2 c}}}{2}
$$

then, for all $\delta$ complex numbers, Re $\delta \geq R e \gamma$, the integral operator $\mathcal{C}_{n}$, given by (2) is in the class $\mathcal{S}$.

Proof. Let us define the function

$$
H_{n}(z)=\int_{0}^{z} \prod_{i=1}^{n}\left[\left(\frac{f_{i}(t)}{t} e^{g_{i}(t)}\right)^{\alpha_{i}-1}\left(h_{i}{ }^{\prime}(t)\right)^{\beta_{i}}\left(\frac{h_{i}(t)}{t}\right)^{\gamma_{i}}\right] \mathrm{dt},
$$

for $f_{i}, g_{i}, h_{i} \in \mathcal{A}, i=\overline{1, n}$.

The function $H_{n}$ is regular in $\mathbb{U}$ and satisfy the following usual normalization conditions $H_{n}(0)=H_{n}^{\prime}(0)-1=0$.

After we calculate the first-order and second-order derivatives, we obtain

$$
\frac{z H_{n}^{\prime \prime}(z)}{H_{n}^{\prime}(z)}=\sum_{i=1}^{n}\left[\left(\alpha_{i}-1\right)\left(\frac{z f_{i}^{\prime}(z)}{f_{i}(z)}-1+z g_{i}^{\prime}(z)\right)+\beta_{i} \frac{z h_{i}^{\prime \prime}(z)}{h_{i}^{\prime}(z)}+\gamma_{i}\left(\frac{z h_{i}^{\prime}(z)}{h_{i}(z)}-1\right)\right],
$$


for all $z \in \mathbb{U}$.

Therefore

$$
\begin{gathered}
\frac{1-|z|^{2 c}}{c}\left|\frac{z H_{n}^{\prime \prime}(z)}{H_{n}^{\prime}(z)}\right| \leq \frac{1-|z|^{2 c}}{c} \sum_{i=1}^{n}\left[\left|\alpha_{i}-1\right|\left(\left|\frac{z f_{i}^{\prime}(z)}{f_{i}(z)}-1\right|+\left|z g_{i}^{\prime}(z)\right|\right)\right]+ \\
+\frac{1-|z|^{2 c}}{c} \sum_{i=1}^{n}\left[\left|\beta_{i}\right|\left|\frac{z h_{i}^{\prime \prime}(z)}{h_{i}^{\prime}(z)}\right|+\left|\gamma_{i}\right|\left|\frac{z h_{i}^{\prime}(z)}{h_{i}(z)}-1\right|\right],
\end{gathered}
$$

for all $z \in \mathbb{U}$.

Then, we obtain

$$
\begin{gathered}
\frac{1-|z|^{2 c}}{c}\left|\frac{z H_{n}^{\prime \prime}(z)}{H_{n}^{\prime}(z)}\right| \leq \frac{1-|z|^{2 c}}{c} \sum_{i=1}^{n}\left[\left|\alpha_{i}-1\right|\left(\left|\frac{z f_{i}^{\prime}(z)}{f_{i}(z)}-1\right|+\left|\frac{z g_{i}{ }^{\prime}(z)}{g_{i}(z)}\right|\left|g_{i}(z)\right|\right)\right]+ \\
+\frac{1-|z|^{2 c}}{c} \sum_{i=1}^{n}\left[\left|\beta_{i}\right|\left|\frac{z h_{i}^{\prime \prime}(z)}{h_{i}^{\prime}(z)}\right|+\left|\gamma_{i}\right|\left|\frac{z h_{i}^{\prime}(z)}{h_{i}(z)}-1\right|\right],
\end{gathered}
$$

for all $z \in \mathbb{U}$.

By applying the General Schwarz Lemma to the functions $f_{i}, g_{i}, i=\overline{1, n}$ we obtain

$$
\begin{aligned}
& \left|\frac{z f_{i}^{\prime}(z)}{f_{i}(z)}-1\right| \leq M_{i}|z|, \quad\left|g_{i}(z)\right| \leq N_{i}|z|, \\
& \left|\frac{z h_{i}^{\prime}(z)}{h_{i}(z)}-1\right| \leq P_{i}|z|, \quad\left|\frac{z h_{i}^{\prime \prime}(z)}{h_{i}^{\prime}(z)}\right| \leq Q_{i}|z|,
\end{aligned}
$$

for all $z \in \mathbb{U}, i=\overline{1, n}$.

Using these inequalities from (4) we have

(5) $\frac{1-|z|^{2 c}}{c}\left|\frac{z H_{n}^{\prime \prime}(z)}{H_{n}^{\prime}(z)}\right| \leq \frac{1-|z|^{2 c}}{c}|z| \sum_{i=1}^{n}\left[\left|\alpha_{i}-1\right|\left(M_{i}+N_{i}\right)+\left|\beta_{i}\right| P_{i}+\left|\gamma_{i}\right| Q_{i}\right]$,

for all $z \in \mathbb{U}$.

Since

$$
\max _{|z| \leq 1} \frac{\left(1-|z|^{2 c}\right)|z|}{c}=\frac{2}{(2 c+1)^{\frac{2 c+1}{2 c}}}
$$

from (5) we obtain

$$
\frac{1-|z|^{2 c}}{c}\left|\frac{z H_{n}^{\prime \prime}(z)}{H_{n}^{\prime}(z)}\right| \leq \frac{2}{(2 c+1)^{\frac{2 c+1}{2 c}}} \sum_{i=1}^{n}\left[\left|\alpha_{i}-1\right|\left(M_{i}+N_{i}\right)+\left|\beta_{i}\right| P_{i}+\left|\gamma_{i}\right| Q_{i}\right],
$$

and hence, by (3) we have

$$
\frac{1-|z|^{2 c}}{c}\left|\frac{z H_{n}^{\prime \prime}(z)}{H_{n}^{\prime}(z)}\right| \leq \frac{2}{(2 c+1)^{\frac{2 c+1}{2 c}}} \frac{(2 c+1)^{\frac{2 c+1}{2 c}}}{2}=1
$$


for all $z \in \mathbb{U}$.

So,

$$
\frac{1-|z|^{2 c}}{c}\left|\frac{z H_{n}^{\prime \prime}(z)}{H_{n}^{\prime}(z)}\right| \leq 1
$$

and using (6), by Lemma 1.2, it results that the integral operator $\mathcal{C}_{n}$, given by $(2)$ is in the class $\mathcal{S}$.

Theorem 2.2. Let $\gamma, \alpha_{i}, \beta_{i}, \gamma_{i}$ be complex numbers, $c=$ Re $\gamma>0$ and $f_{i}, h_{i} \in \mathcal{S}$, $h_{i}{ }^{\prime} \in \mathcal{P}, g_{i} \in \mathcal{R}, f_{i}(z)=z+a_{2 i} z^{2}+a_{3 i} z^{3}+\ldots ., g_{i}(z)=z+b_{2 i} z^{2}+b_{3 i} z^{3}+\ldots$. $h_{i}(z)=z+c_{2 i} z^{2}+c_{3 i} z^{3}+\ldots ., i=\overline{1, n}$. If

$$
4 \sum_{i=1}^{n}\left|\alpha_{i}-1\right|+\sum_{i=1}^{n}\left|\beta_{i}\right|+2 \sum_{i=1}^{n}\left|\gamma_{i}\right| \leq \frac{c}{2}, \quad \text { for } \quad 0<c<1
$$

or

$$
4 \sum_{i=1}^{n}\left|\alpha_{i}-1\right|+\sum_{i=1}^{n}\left|\beta_{i}\right|+2 \sum_{i=1}^{n}\left|\gamma_{i}\right| \leq \frac{1}{2}, \quad \text { for } \quad c \geq 1
$$

then, for any complex numbers $\delta, R e \delta \geq c$, the integral operator $\mathcal{C}_{n}$ defined by (2) is in the class $\mathcal{S}$.

Proof. From the proof of Theorem 2.1, we have:

$$
\begin{gathered}
\frac{1-|z|^{2 c}}{c}\left|\frac{z H_{n}^{\prime \prime}(z)}{H_{n}^{\prime}(z)}\right| \leq \frac{1-|z|^{2 c}}{c} \sum_{i=1}^{n}\left[\left|\alpha_{i}-1\right|\left(\left|\frac{z f_{i}^{\prime}(z)}{f_{i}(z)}-1\right|+\left|z g_{i}^{\prime}(z)\right|\right)\right]+ \\
+\frac{1-|z|^{2 c}}{c} \sum_{i=1}^{n}\left[\left|\beta_{i}\right|\left|\frac{z h_{i}^{\prime \prime}(z)}{h_{i}^{\prime}(z)}\right|+\left|\gamma_{i}\right|\left|\frac{z h_{i}^{\prime}(z)}{h_{i}(z)}-1\right|\right],
\end{gathered}
$$

for all $z \in \mathbb{U}$.

$$
\begin{gathered}
\frac{1-|z|^{2 c}}{c}\left|\frac{z H_{n}^{\prime \prime}(z)}{H_{n}^{\prime}(z)}\right| \leq \frac{1-|z|^{2 c}}{c} \sum_{i=1}^{n}\left[\left|\alpha_{i}-1\right|\left(\left|\frac{z f_{i}^{\prime}(z)}{f_{i}(z)}\right|+1+\left|z g_{i}^{\prime}(z)\right|\right)\right]+ \\
+\frac{1-|z|^{2 c}}{c} \sum_{i=1}^{n}\left[\left|\beta_{i}\right|\left|\frac{z h_{i}^{\prime \prime}(z)}{h_{i}^{\prime}(z)}\right|+\left|\gamma_{i}\right|\left(\left|\frac{z h_{i}^{\prime}(z)}{h_{i}(z)}\right|+1\right)\right],
\end{gathered}
$$

for all $z \in \mathbb{U}$.

Since $f_{i}, h_{i} \in \mathcal{S}$ we have

$$
\left|\frac{z f_{i}^{\prime}(z)}{f_{i}(z)}\right| \leq \frac{1+|z|}{1-|z|}, \quad\left|\frac{z h_{i}^{\prime}(z)}{h_{i}(z)}\right| \leq \frac{1+|z|}{1-|z|}
$$

for all $z \in \mathbb{U}, i=\overline{1, n}$. 
For $h_{i}{ }^{\prime} \in \mathcal{P}$ we have

$$
\left|\frac{z h_{i}^{\prime \prime}(z)}{h_{i}^{\prime}(z)}\right| \leq \frac{2|z|}{1-|z|^{2}}
$$

for all $z \in \mathcal{U}, i=\overline{1, n}$.

For $g_{i} \in \mathcal{R}$ we have $g_{i}{ }^{\prime} \in \mathcal{P}$ and

$$
\left|g_{i}{ }^{\prime}(z)\right| \leq \frac{1+|z|}{1-|z|}
$$

Using these relations we get

$$
\begin{gathered}
\frac{1-|z|^{2 c}}{c}\left|\frac{z H_{n}^{\prime \prime}(z)}{H_{n}^{\prime}(z)}\right| \leq \frac{1-|z|^{2 c}}{c}\left[\left(\frac{1+|z|}{1-|z|}+1+\frac{|z|(1+|z|)}{1-|z|}\right) \sum_{i=1}^{n}\left|\alpha_{i}-1\right|\right]+ \\
+\frac{1-|z|^{2 c}}{c} \frac{2|z|}{1-|z|^{2}} \sum_{i=1}^{n}\left|\beta_{i}\right|+\frac{1-|z|^{2 c}}{c}\left[\left(\frac{1+|z|}{1-|z|}+1\right) \sum_{i=1}^{n}\left|\gamma_{i}\right|\right] \leq \\
\leq \frac{1-|z|^{2 c}}{c}\left(\frac{2}{1-|z|}+\frac{|z|(1+|z|)}{1-|z|}\right) \sum_{i=1}^{n}\left|\alpha_{i}-1\right|+ \\
+\frac{1-|z|^{2 c}}{c} \frac{2|z|}{1-|z|^{2}} \sum_{i=1}^{n}\left|\beta_{i}\right|+\frac{1-|z|^{2 c}}{c} \frac{2}{1-|z|} \sum_{i=1}^{n}\left|\gamma_{i}\right|
\end{gathered}
$$

for all $z \in \mathbb{U}$.

For $0<c<1$, we have $1-|z|^{2 c} \leq 1-|z|^{2}, z \in \mathbb{U}$ and by (9) we obtain

$$
\frac{1-|z|^{2 c}}{c}\left|\frac{z H_{n}^{\prime \prime}(z)}{H_{n}^{\prime}(z)}\right| \leq\left(\frac{4}{c}+\frac{4}{c}\right) \sum_{i=1}^{n}\left|\alpha_{i}-1\right|+\frac{2}{c} \sum_{i=1}^{n}\left|\beta_{i}\right|+\frac{4}{c} \sum_{i=1}^{n}\left|\gamma_{i}\right|,
$$

for all $z \in \mathbb{U}$.

From (7) and (10) we have

$$
\frac{1-|z|^{2 c}}{c}\left|\frac{z H_{n}^{\prime \prime}(z)}{H_{n}^{\prime}(z)}\right| \leq 1
$$

for all $z \in \mathbb{U}$ and $0<c<1$.

For $c \geq 1$ we have $\frac{1-|z|^{i c}}{c} \leq 1-|z|^{2}$, for all $z \in \mathcal{U}$ and by (9) we obtain

$$
\frac{1-|z|^{2 c}}{c}\left|\frac{z H_{n}^{\prime \prime}(z)}{H_{n}^{\prime}(z)}\right| \leq(4+4) \sum_{i=1}^{n}\left|\alpha_{i}-1\right|+2 \sum_{i=1}^{n}\left|\beta_{i}\right|+4 \sum_{i=1}^{n}\left|\gamma_{i}\right|,
$$

for all $z \in \mathbb{U}$ and $c \geq 1$.

From (8) and (12) we obtain

$$
\frac{1-|z|^{2 c}}{c}\left|\frac{z H_{n}^{\prime \prime}(z)}{H_{n}^{\prime}(z)}\right| \leq 1
$$


for all $z \in \mathbb{U}$ and $c \geq 1$.

And by (11), (13) and Lemma 1.2 it results that the integral operator $\mathcal{C}_{n}$, defined by (2) is in the class $\mathcal{S}$.

Theorem 2.3. Let $\alpha$ be complex number, Re $\alpha>0, i=\overline{1, n}, M_{i}, N_{i}, P_{i}$ are real positive numbers and $f_{i}, g_{i}, h_{i} \in \mathcal{A}, f_{i}(z)=z+a_{2 i} z^{2}+a_{3 i} z^{3}+\ldots ., g_{i}(z)=z+b_{2 i} z^{2}+$ $b_{3 i} z^{3}+\ldots, h_{i}(z)=z+c_{2 i} z^{2}+c_{3 i} z^{3}+\ldots ., i=\overline{1, n}$. If

$$
\begin{gathered}
\left|\frac{z f_{i}^{\prime}(z)}{f_{i}(z)}\right| \leq M_{i}, \quad\left|g_{i}(z)\right| \leq N_{i}, \quad\left|\frac{z h_{i}^{\prime \prime}(z)}{h_{i}^{\prime}(z)}\right| \leq 1, \\
\left|\frac{z h_{i}^{\prime}(z)}{h_{i}(z)}-1\right| \leq P_{i}, \quad\left|\frac{z^{2} g_{i}^{\prime}(z)}{\left[g_{i}(z)\right]^{2}}-1\right|<1,
\end{gathered}
$$

for all $z \in \mathbb{U}, i=\overline{1, n}$ and

$$
|c| \leq 1-\left|\frac{\alpha-1}{\alpha}\right|\left(M_{i}+2 N_{i}^{2}+P_{i}+3\right), \quad c \in \mathbb{C}, \quad c \neq-1,
$$

then, the integral operator $\mathcal{C}_{n}^{*}$, given by

$$
\mathcal{C}_{n}^{*}(z)=\left[\alpha \int_{0}^{z} t^{\alpha-1} \prod_{i=1}^{n}\left(\frac{f_{i}(t)}{t} e^{g_{i}(t)} h_{i}{ }^{\prime}(t) \frac{\left.h_{i}(t)\right)}{t}\right)^{\alpha-1} d t\right]^{\frac{1}{\alpha}}
$$

is in the class $\mathcal{S}$.

Proof. Let us consider the function

$$
H_{n}(z)=\int_{0}^{z} \prod_{i=1}^{n}\left(\frac{f_{i}(t)}{t} e^{g_{i}(t)} h_{i}{ }^{\prime}(t) \frac{\left.h_{i}(t)\right)}{t}\right)^{\alpha-1} \mathrm{dt},
$$

for $f_{i}, g_{i}, h_{i} \in \mathcal{A}, i=\overline{1, n}$. The function $H_{n}$ is regular in $\mathbb{U}$.

Also, a simple computation yields

$$
\frac{z H_{n}^{\prime \prime}(z)}{H_{n}^{\prime}(z)}=\sum_{i=1}^{n}\left[(\alpha-1)\left(\frac{z f_{i}^{\prime}(z)}{f_{i}(z)}-1+z g_{i}^{\prime}(z)+\frac{z h_{i}^{\prime \prime}(z)}{h_{i}^{\prime}(z)}+\frac{z h_{i}^{\prime}(z)}{h_{i}(z)}-1\right)\right],
$$

for all $z \in \mathbb{U}$.

Therefore

$$
\begin{gathered}
\left.|c| z\right|^{2 \alpha}+\left(1-|z|^{2 \alpha}\right) \frac{z H_{n}^{\prime \prime}(z)}{\alpha H_{n}^{\prime}(z)} \mid= \\
=\left.|c| z\right|^{2 \alpha}+\left(1-|z|^{2 \alpha}\right) \frac{1}{\alpha} \sum_{i=1}^{n}\left[(\alpha-1)\left(\frac{z f_{i}^{\prime}(z)}{f_{i}(z)}-1+z g_{i}^{\prime}(z)\right)\right] \mid \\
+\left.|c| z\right|^{2 \alpha}+\left(1-|z|^{2 \alpha}\right) \frac{1}{\alpha} \sum_{i=1}^{n}\left[(\alpha-1)\left(\frac{z h_{i}^{\prime \prime}(z)}{h_{i}^{\prime}(z)}+\frac{z h_{i}^{\prime}(z)}{h_{i}(z)}-1\right)\right] \mid
\end{gathered}
$$


for all $z \in \mathbb{U}$.

Then, we obtain

$$
\left.|c| z\right|^{2 \alpha}+\left(1-|z|^{2 \alpha}\right) \frac{z H_{n}^{\prime \prime}(z)}{\alpha H_{n}^{\prime}(z)} \mid \leq
$$

$$
\leq|c|+\left|\frac{\alpha-1}{\alpha}\right|\left[\left(\left|\frac{z f_{i}^{\prime}(z)}{f_{i}(z)}\right|+1\right)+\left|\frac{z^{2} g_{i}(z)}{\left[g_{i}(z)\right]^{2}}\right|\left|\frac{\left[g_{i}(z)\right]^{2}}{z}\right|+\left|\frac{z h_{i}^{\prime \prime}(z)}{h_{i}^{\prime}(z)}\right|+\left(\left|\frac{z h_{i}^{\prime}(z)}{h_{i}(z)}\right|+1\right)\right],
$$

for all $z \in \mathbb{U}$.

By applying the General Schwarz Lemma to the functions $g_{i}, i=\overline{1, n}$ we obtain

$$
\left|g_{i}(z)\right| \leq N_{i}|z|
$$

for all $z \in \mathbb{U}, i=\overline{1, n}$.

Using these inequalities from (16) we have

$$
\left.|c| z\right|^{2 \alpha}+\left(1-|z|^{2 \alpha}\right) \frac{z H_{n}^{\prime \prime}(z)}{\alpha H_{n}^{\prime}(z)}|\leq| c|+| \frac{\alpha-1}{\alpha} \mid\left[M_{i}+1+2 N_{i}^{2}+1+P_{i}+1\right],
$$

for all $z \in \mathbb{U}$.

So

$$
\left.|c| z\right|^{2 \alpha}+\left(1-|z|^{2 \alpha}\right) \frac{z H_{n}^{\prime \prime}(z)}{\alpha H_{n}^{\prime}(z)}|\leq| c|+| \frac{\alpha-1}{\alpha} \mid\left[M_{i}+2 N_{i}^{2}+P_{i}+3\right],
$$

and hence, by inequality (14) we have

$$
\left.|c| z\right|^{2 \alpha}+\left(1-|z|^{2 \alpha}\right) \frac{z H_{n}^{\prime \prime}(z)}{H_{n}^{\prime}(z)} \mid \leq 1
$$

for all $z \in \mathbb{U}$.

Applying Lemma 1.3, we conclude that the integral operator $\mathcal{C}_{n}^{*}$, given by $(15)$ is in the class $\mathcal{S}$.

\section{Corollaries and consequences}

First of all, upon setting $\delta=1$ in Theorem 2.1, we immediately arrive at the following corollary:

Corollary 3.1. Let $\gamma, \alpha_{i}, \beta_{i}, \gamma_{i}$ be complex numbers, $0<$ Re $\gamma \leq 1, M_{i}, N_{i}, P_{i}, Q_{i}$ are real positive numbers and $f_{i}, g_{i}, h_{i} \in \mathcal{A}, f_{i}(z)=z+a_{2 i} z^{2}+a_{3 i} z^{3}+\ldots ., g_{i}(z)=$ $z+b_{2 i} z^{2}+b_{3 i} z^{3}+\ldots ., h_{i}(z)=z+c_{2 i} z^{2}+c_{3 i} z^{3}+\ldots ., i=\overline{1, n}$. If

$$
\begin{gathered}
\left|\frac{z f_{i}^{\prime}(z)}{f_{i}(z)}-1\right| \leq M_{i}, \quad\left|g_{i}(z)\right| \leq N_{i}, \quad\left|\frac{z h_{i}^{\prime \prime}(z)}{h_{i}^{\prime}(z)}\right| \leq P_{i}, \\
\left|\frac{z h_{i}^{\prime}(z)}{h_{i}(z)}-1\right| \leq Q_{i}, \quad\left|\frac{z g_{i}^{\prime}(z)}{g_{i}(z)}\right| \leq 1,
\end{gathered}
$$


for all $z \in \mathbb{U}, i=\overline{1, n}$ and

$$
\sum_{i=1}^{n}\left[\left|\alpha_{i}-1\right|\left(M_{i}+N_{i}\right)+\left|\beta_{i}\right| P_{i}+\left|\gamma_{i}\right| Q_{i}\right] \leq \frac{(2 c+1)^{\frac{2 c+1}{2 c}}}{2},
$$

then, the integral operator $\mathcal{K}_{n}$ defined by

$$
\mathcal{K}_{n}(z)=\int_{0}^{z} \coprod_{i=1}^{n}\left[\left(\frac{f_{i}(t)}{t} e^{g_{i}(t)}\right)^{\alpha_{i}-1}\left(h_{i}{ }^{\prime}(t)\right)^{\beta_{i}}\left(\frac{\left.h_{i}(t)\right)}{t}\right)^{\gamma_{i}}\right] d t
$$

is in the class $\mathcal{S}$.

If we consider $\delta=1$ and $\gamma_{i}=0$ in Theorem 2.1, obtain the next corollary:

Corollary 3.2. Let $\gamma, \alpha_{i}, \beta_{i}$ be complex numbers, $0<$ Re $\gamma \leq 1, c=\operatorname{Re} \gamma, M_{i}, N_{i}, P_{i}$ are real positive numbers and $f_{i}, g_{i}, h_{i} \in \mathcal{A}, f_{i}(z)=z+a_{2 i} z^{2}+a_{3 i} z^{3}+\ldots ., g_{i}(z)=$ $z+b_{2 i} z^{2}+b_{3 i} z^{3}+\ldots ., h_{i}(z)=z+c_{2 i} z^{2}+c_{3 i} z^{3}+\ldots ., i=\overline{1, n}$. If

$$
\left|\frac{z f_{i}^{\prime}(z)}{f_{i}(z)}-1\right| \leq M_{i}, \quad\left|g_{i}(z)\right| \leq N_{i}, \quad\left|\frac{z h_{i}^{\prime \prime}(z)}{h_{i}^{\prime}(z)}\right| \leq P_{i}, \quad\left|\frac{z g_{i}^{\prime}(z)}{g_{i}(z)}\right| \leq 1,
$$

for all $z \in \mathbb{U}, i=\overline{1, n}$ and

$$
\sum_{i=1}^{n}\left[\left|\alpha_{i}-1\right|\left(M_{i}+N_{i}\right)+\left|\beta_{i}\right| P_{i}\right] \leq \frac{(2 c+1)^{\frac{2 c+1}{2 c}}}{2},
$$

then, the integral operator $\mathcal{T}_{n}$ defined by

$$
\mathcal{T}_{n}(z)=\int_{0}^{z} \coprod_{i=1}^{n}\left[\left(\frac{f_{i}(t)}{t} e^{g_{i}(t)}\right)^{\alpha_{i}-1}\left(h_{i}{ }^{\prime}(t)\right)^{\beta_{i}}\right] d t,
$$

is in the class $\mathcal{S}$.

Remark 3.3. In the integral operator given by (19) if we take $\gamma_{i}=0$, we obtain known result proven in [19].

If we consider $\delta=1$ and $\beta_{i}=0$ in Theorem 2.1, obtain the next corollary:

Corollary 3.4. Let $\gamma, \alpha_{i}, \gamma_{i}$ be complex numbers, $0<$ Re $\gamma \leq 1, c=\operatorname{Re} \gamma, M_{i}, N_{i}, Q_{i}$ are real positive numbers and $f_{i}, g_{i}, h_{i} \in \mathcal{A}, f_{i}(z)=z+a_{2 i} z^{2}+a_{3 i} z^{3}+\ldots ., g_{i}(z)=$ $z+b_{2 i} z^{2}+b_{3 i} z^{3}+\ldots ., h_{i}(z)=z+c_{2 i} z^{2}+c_{3 i} z^{3}+\ldots ., i=\overline{1, n}$. If

$$
\left|\frac{z f_{i}^{\prime}(z)}{f_{i}(z)}-1\right| \leq M_{i}, \quad\left|g_{i}(z)\right| \leq N_{i}, \quad\left|\frac{z h_{i}^{\prime}(z)}{h_{i}(z)}-1\right| \leq Q_{i}, \quad\left|\frac{z g_{i}^{\prime}(z)}{g_{i}(z)}\right| \leq 1,
$$

for all $z \in \mathbb{U}, i=\overline{1, n}$ and

$$
\sum_{i=1}^{n}\left[\left|\alpha_{i}-1\right|\left(M_{i}+N_{i}\right)+\left|\gamma_{i}\right| Q_{i}\right] \leq \frac{(2 c+1)^{\frac{2 c+1}{2 c}}}{2},
$$


then, the integral operator $\mathcal{R}_{n}$ defined by

$$
\mathcal{R}_{n}(z)=\int_{0}^{z} \coprod_{i=1}^{n}\left[\left(\frac{f_{i}(t)}{t} e^{g_{i}(t)}\right)^{\alpha_{i}-1}\left(\frac{\left.h_{i}(t)\right)}{t}\right)^{\gamma_{i}}\right] d t,
$$

is in the class $\mathcal{S}$.

Remark 3.5. Putting $\beta_{i}=0$ in (20) we obtain the integral operator introduced in [19].

If we consider $\delta=1$ and $\alpha_{i}=0$ in Theorem 2.1, obtain the next corollary:

Corollary 3.6. Let $\gamma, \beta_{i}, \gamma_{i}$ be complex numbers, $0<\operatorname{Re} \gamma \leq 1, c=\operatorname{Re} \gamma, P_{i}, Q_{i}$ are real positive numbers and $h_{i} \in \mathcal{A}, h_{i}(z)=z+b_{2 i} z^{2}+b_{3 i} z^{3}+\ldots ., i=\overline{1, n}$. If

$$
\left|\frac{z h_{i}^{\prime \prime}(z)}{h_{i}^{\prime}(z)}\right| \leq P_{i}, \quad\left|\frac{z h_{i}^{\prime}(z)}{h_{i}(z)}-1\right| \leq Q_{i},
$$

for all $z \in \mathbb{U}, i=\overline{1, n}$ and

$$
\sum_{i=1}^{n}\left[\left|\beta_{i}\right| P_{i}+\left|\gamma_{i}\right| Q_{i}\right] \leq \frac{(2 c+1)^{\frac{2 c+1}{2 c}}}{2},
$$

then, the integral operator $\mathcal{I}_{n}$ defined by

$$
\mathcal{I}_{n}(z)=\int_{0}^{z} \coprod_{i=1}^{n}\left[\left(h_{i}{ }^{\prime}(t)\right)^{\beta_{i}}\left(\frac{\left.h_{i}(t)\right)}{t}\right)^{\gamma_{i}}\right] d t,
$$

is in the class $\mathcal{S}$.

Remark 3.7. The integral operator from Corollary 3.6, given by (21) is a known result proven in [5].

If we consider $\delta=1, \beta_{i}=0$ and $\gamma_{i}=0$ in Theorem 2.1, obtain the next corollary:

Corollary 3.8. Let $\gamma, \alpha_{i}$ be complex numbers, $0<\operatorname{Re} \gamma \leq 1, c=\operatorname{Re}, M_{i}, N_{i}$ are real positive numbers and $f_{i}, g_{i} \in \mathcal{A}, f_{i}(z)=z+a_{2 i} z^{2}+a_{3 i} z^{3}+\ldots ., g_{i}(z)=$ $z+b_{2 i} z^{2}+b_{3 i} z^{3}+\ldots ., i=\overline{1, n}$. If

$$
\left|\frac{z f_{i}^{\prime}(z)}{f_{i}(z)}-1\right| \leq M_{i}, \quad\left|g_{i}(z)\right| \leq N_{i}, \quad\left|\frac{z g_{i}^{\prime}(z)}{g_{i}(z)}\right| \leq 1,
$$

for all $z \in \mathbb{U}, i=\overline{1, n}$ and

$$
\sum_{i=1}^{n}\left[\left|\alpha_{i}-1\right|\left(M_{i}+N_{i}\right)\right] \leq \frac{(2 c+1)^{\frac{2 c+1}{2 c}}}{2},
$$

then, the integral operator $\mathcal{I}_{n}$ defined by

$$
\mathcal{I}_{n}(z)=\int_{0}^{z} \coprod_{i=1}^{n}\left[\left(\frac{f_{i}(t)}{t} e^{g_{i}(t)}\right)^{\alpha_{i}-1}\right] d t,
$$

is in the class $\mathcal{S}$. 
Remark 3.9. This integral operator given by (22) was defined in [19].

If we consider $\delta=1, \alpha_{i}=0$ and $\gamma_{i}=0$ in Theorem 2.1, obtain the next corollary:

Corollary 3.10. Let $\gamma, \beta_{i}$ be complex numbers, $0<$ Re $\gamma \leq 1, c=$ Re $\gamma, P_{i}$ are real positive numbers and $h_{i} \in \mathcal{A}, h_{i}(z)=z+c_{2 i} z^{2}+c_{3 i} z^{3}+\ldots ., i=\overline{1, n}$. If

$$
\left|\frac{z h_{i}^{\prime \prime}(z)}{h_{i}^{\prime}(z)}\right| \leq P_{i}
$$

for all $z \in \mathbb{U}, i=\overline{1, n}$ and

$$
\sum_{i=1}^{n}\left[\left|\beta_{i}\right| P_{i}\right] \leq \frac{(2 c+1)^{\frac{2 c+1}{2 c}}}{2},
$$

then, the integral operator $\mathcal{I}_{n}$ defined by

$$
\mathcal{I}_{n}(z)=\int_{0}^{z} \coprod_{i=1}^{n}\left(h_{i}{ }^{\prime}(t)\right)^{\beta_{i}} d t
$$

is in the class $\mathcal{S}$.

Remark 3.11. The integral operator from Corollary 3.10, given by (23) is another known result proven in [4].

If we consider $\delta=1, \alpha_{i}=0$ and $\beta_{i}=0$ in Theorem 2.1, obtain the next corollary:

Corollary 3.12. Let $\gamma, \gamma_{i}$ be complex numbers, $0<$ Re $\gamma \leq 1, c=$ Re $\gamma, Q_{i}$ are real positive numbers and $h_{i} \in \mathcal{A}, h_{i}(z)=z+c_{2 i} z^{2}+c_{3 i} z^{3}+\ldots, i=\overline{1, n}$. If

$$
\left|\frac{z h_{i}^{\prime}(z)}{h_{i}(z)}-1\right| \leq Q_{i}
$$

for all $z \in \mathbb{U}, i=\overline{1, n}$ and

$$
\sum_{i=1}^{n}\left[\left|\gamma_{i}\right| Q_{i}\right] \leq \frac{(2 c+1)^{\frac{2 c+1}{2 c}}}{2}
$$

then, the integral operator $\mathcal{I}_{n}$ defined by

$$
\mathcal{I}_{n}(z)=\int_{0}^{z} \coprod_{i=1}^{n}\left(\frac{\left.h_{i}(t)\right)}{t}\right)^{\gamma_{i}} d t
$$

is in the class $\mathcal{S}$.

Remark 3.13. This integral operator given by (24) is a well know result proven in [2]. 
If we consider $n=1, \delta=\gamma=\alpha$ and $\alpha_{i}-1=\beta_{i}=\gamma_{i}$ in Theorem 2.1, obtain the next corollary:

Corollary 3.14. Let $\alpha$ be complex number, Re $\alpha>0, M, N, P, Q$ are real positive numbers and $f, g, h \in \mathcal{A}, f(z)=z+a_{2} z^{2}+a_{3} z^{3}+\ldots, g(z)=z+b_{2} z^{2}+b_{3} z^{3}+\ldots$, $h(z)=z+c_{2} z^{2}+c_{3} z^{3}+\ldots$. If

$\left|\frac{z f^{\prime}(z)}{f(z)}-1\right| \leq M, \quad|g(z)| \leq N, \quad\left|\frac{z h^{\prime \prime}(z)}{h^{\prime}(z)}\right| \leq P, \quad\left|\frac{z h^{\prime}(z)}{h(z)}-1\right| \leq Q, \quad\left|\frac{z g^{\prime}(z)}{g(z)}\right| \leq 1$,

for all $z \in \mathbb{U}$ and

$$
|\alpha-1|(M+N+P+Q) \leq \frac{(2 \operatorname{Re} \alpha+1)^{\frac{2 R e e+1}{2 R e \alpha}}}{2},
$$

then, the integral operator $\mathcal{C}$ defined by

$$
\mathcal{C}(z)=\left\{\alpha \int_{0}^{z}\left[f(t) e^{g(t)} h^{\prime}(t) \frac{h(t))}{t}\right]^{\alpha-1} d t\right\}^{\frac{1}{\alpha}}
$$

is in the class $\mathcal{S}$.

\section{References}

[1] C. L. Aldea, V. Pescar, Univalence Criteria for a general integral operator, Transilvania University of Brasov, vol. 10, no. 2, 2017, 19-30.

[2] D. Breaz, N. Breaz, Two Integral Operators, Studia Univ."Babes-Bolyai", ClujNapoca, Mathematica, vol. 47, no. 3, 2002, 13-21.

[3] D. Breaz, N. Breaz, H. M. Srivastava, An extension of the univalent condition for a family of integral operators, Appl. Math. Lett., vol. 22, no. 3, 2009, 41-44.

[4] D. Breaz, S. Owa, N. Breaz, A new integral univalent operator, Acta Universitatis Apulensis, vol. 16, 2008.

[5] B. A. Frasin, Order of convexity and univalence of general integral operator, Journal of the Franklin, vol. 348, 2011, 1012-1019.

[6] B. A. Frasin, Sufficient conditions for the univalence of an integral operator, Asia Pacific Journal of Mathematics, vol.5, no.1, 2018, 85-91.

[7] I. J. Kim, E. P. Merkes, On an integral of powers of a spirallike function, Kyungpook Math. J., vol. 12, no. 2, 1972, 249-253.

[8] O. Mayer, The Functions Theory of the One Variable Complex, Acad. Ed., Bucuresti, Romania, 1981, 101-117. 
[9] H. Oversea, Integral operators of Bazilvic type, Bull. Math. Bucuresti, vol. 37, 1993, 115-125.

[10] S. Ozaki, M. Nunokawa, The Schwarzian derivative and univalent functions, Proceedings of the American Mathematical Society, Mathematics, vol. 33, 1972, 392-394.

[11] N. N. Pascu, An improvement of Beker's univalence criterion, Proceedings of the Commemorative Session Simion Stoilov, University of Braso, 1987, 43-48.

[12] N. N. Pascu, V. Pescar, On the integral operators Kim-Merkes and Pfaltzgraff, Mathematica, UBB, Cluj-Napoca, vol. 32, no. 2, 1990, 185-192.

[13] V. Pescar, New univalence criteria for some integral operators, Studia Univ."Babes-Bolyai", Cluj-Napoca, Mathematica, vol. 59, no. 2, 2014, 185-192.

[14] V. Pescar, A new generalization of Ahlfors's and Becker's criterion of univalence, Bulletin of Malaysian Mathematical Society, vol. 19, no.2, 1996, 53-54.

[15] V. Pescar, Univalence criteria of certain integral operators, Acta Ciencia Indica, Mathematics, vol. 29, no. 1, 2003, 135-138.

[16] V. Pescar, On the univalence of some integral operators, General Mathematics, vol. 14 , no. $2,2006,77-84$.

[17] V. Pescar, S. Owa, Univalence of certain integral operators, Int. J. Math. Math. Sci., vol. 23, 2000, 697-701.

[18] J. Pfaltzgraff, Univalence of the integral of $\left(f^{\prime}(z)\right)^{\lambda}$, Bull. London Math. Soc., vol. 7 , no. 3, 1975, 254-256.

[19] L. Stanciu, The Univalence conditions of some integral operators, Abstract and Applied Analysis, ID 924645, 2012, 9 pages.

[20] L. F. Stanciu, D. Breaz, H. M. Srivastava, Some criteria for univalence of a certain integral operator, Novi Sad J. Math. vol. 43, no. 2, 2013, 51-57.

[21] P. T. Mocanu, T. Bulboaca, G. S. Salagean, Teoria geometrica a functiilor univalente, Casa Cartii de Stiinta, Cluj Napoca, 1999, 77-81.

\section{Camelia Bărbatu}

"Babeş-Bolyai" University

Faculty of Mathematics and Computer Sciences

Department of Mathematics

1, Kogălniceanu Street, 400084 Cluj-Napoca, Romania

e-mail: camipode@yahoo.com 


\section{Daniel Breaz}

"1 Decembrie 1918" University of Alba Iulia

Faculty of Science

Department of Mathematics

5, Gabriel Bethlen Street, 510009 Alba-Iulia, Romania

e-mail: dbreaz@uab.ro 